University of Nebraska - Lincoln

DigitalCommons@University of Nebraska - Lincoln

2006

\title{
Spontaneous Facial Motility in Infancy: A 3D Kinematic Analysis
}

Jordan R. Green

University of Nebraska-Lincoln, jgreen4@unl.edu

Erin M. Wilson

University of Wisconsin-Madison

Follow this and additional works at: https://digitalcommons.unl.edu/specedfacpub

Part of the Special Education and Teaching Commons

Green, Jordan R. and Wilson, Erin M., "Spontaneous Facial Motility in Infancy: A 3D Kinematic Analysis" (2006). Special Education and Communication Disorders Faculty Publications. 46.

https://digitalcommons.unl.edu/specedfacpub/46

This Article is brought to you for free and open access by the Department of Special Education and Communication Disorders at DigitalCommons@University of Nebraska - Lincoln. It has been accepted for inclusion in Special Education and Communication Disorders Faculty Publications by an authorized administrator of DigitalCommons@University of Nebraska - Lincoln. 
Published in Developmental Psychobiology 48 (2006), pp. 16-28; doi: 10.1002/dev.20112 Copyright @ 2005 Wiley Periodicals, Inc.

Published online in Wiley InterScience http://www.interscience.wiley.com Used by permission.

This research was sponsored by grants from NIH-NIDCD (Contract grant numbers R03DC04643-03 and R01 DC06463-01)

and The Barkley Trust.

Submitted December 14, 2004; accepted August 22, 2005.

\title{
Spontaneous Facial Motility in Infancy: A 3D Kinematic Analysis
}

\author{
Jordan R. Green \\ Department of Special Education and Communication Disorders University of Nebraska-Lincoln, 318 Barkley Center, Lincoln, \\ NE 68583 and Institute for Rehabilitation Science and Engineering, Madonna Rehabilitation Hospital, Lincoln, NE 68583; \\ Corresponding author: email jgreen4@unl.edu

\section{Erin M. Wilson} \\ Department of Communicative Disorders University of Wisconsin-Madison Madison, WI
}

\begin{abstract}
Early spontaneous orofacial movements have rarely been studied experimentally, though the motor experiences gained from these behaviors may influence the development of motor skills emerging for speech. This investigation quantitatively describes developmental changes in silent, spontaneous lip and jaw movements from 1 to 12 months of age using optically based 3D motion capture technology. Twenty-nine typically developing infants at five ages (1, 5, 7, 9, and 12 months) were studied cross-sectionally. Infants exhibited spontaneous facial movements at all ages studied. Several age-related changes were detected in lip and jaw kinematics: the occurrence of spontaneous movements increased, movement speed increased, the duration of movement epochs decreased and movement coupling among different facial regions increased. Additionally, evidence for stereotypic movements was not strong. The present findings suggest that, during the first year of life, early spontaneous facial movements undergo significant developmental change in the direction of skill development for speech.
\end{abstract}

Keywords: motor development, speech development, spontaneous motility, jaw, lips, speech motor control

Prenatal and early postnatal development is marked by spontaneous movement (Bekoff, 2001; Birnholz, Stephens, \& Faria, 1978; de Vries, Visser, \& Prechtl, 1982; Geerdink, Hopkins, Beek, \& Heriza, 1996; Hall \& Oppenheim, 1987; Hamburger, Wenger, \& Oppenheim, 1966; Thelen, 1979). Most of the early investigations of spontaneous motility concluded that these movements serve an adaptive function in early development (Coghill, 1929; Hooker, 1944; Hamburger et al., 1966). Empirical support for this suggestion continues to build with more recent work showing spontaneous movements to facilitate: (1) the formation of anatomic and sensorimotor systems (Fields \& Nelson, 1992; Hall \& Herring, 1990; Kalb \& Hockfield, 1992; Oppenheim, Pittman, Gray, \& Maderdrut, 1978) and (2) the development of motor skills for goal-directed behaviors such as walking (Thelen, 1985).

These findings suggest that by performing spontane- ous movements, the infant provides itself with the motor experience it needs for further anatomic and neurologic development. This view of early motor development is consistent with a conceptual shift within the developmental literature from descriptions that focus on "forward reference" systems (e.g., genetic determination) to those that emphasize the importance of sensorimotor experience in both neuronal and behavioral development (Edelman, 1987; Gottlieb, 1992; Kuo, 1967; Thelen, 1995).

The earliest spontaneous movements are thought to be generated by endogenous neural activity (i.e., autonomous discharge of motor neurons), rather than from activity related to myogenics, reflexogenics, or descending input (Hamburger et al., 1966; Roeder, 1963). During early prenatal development, primitive motor neuron rootlets exhibit phasic spontaneous discharge prior to connecting to their target muscle fibers (Gust, Wright, Pratt, \& Bosma, 
2003) and myotubes contract as soon as they are innervated by motoneurons (Landmesser \& Morris, 1975). In animals, spontaneous activity in the spinal nervous system has been observed to evolve even in the absence of cortical, subcortical, and afferent systems (Hamburger et al., 1966; Roeder, 1963). For example, spontaneous movements develop normally even when the entire dorsal half of the chick lumbosacral spinal cord is removed at 2-2.5 days (Hamburger et al., 1966).

Evidence for the continuity between early spontaneous activity and later appearing motor behaviors has been largely drawn from the following observations: (a) spatial or temporal patterning was similar between spontaneous movements and movements associated with an emerging skill (Bekoff, 1986; Thelen \& Fisher, 1983), (b) developmental changes in spontaneous movements were continuous with those emerging for goal-directed behaviors in the limbs (de Vries et al., 1982), and (c) spontaneous activity in a muscle group predicted the rate of motor development of later appearing skills using those muscles (Thelen, 1979).

If the neural circuits that produce spontaneous limb movement in early infancy are later adapted for walking and grasping, then it is conceivable that spontaneous movements of the lips, tongue, and jaw in early development establish sensorimotor associations that could be used to control the gestures of early speech. Spontaneous movements of the jaw appear as early as 11 weeks of prenatal development (de Vries, Visser, \& Prechtl, 1985). Several investigators have suggested that spontaneous oral movements in the fetus emerge in the following order: jaw opening, jaw closing, tongue movement, and lip movement (see Herring, 1985; Humphrey, 1970, 1971). Jaw opening and closing are also primary features of babble and early speech (Green, Moore, Higashikawa, \& Steeve, 2000).

One untested hypothesis is that, in early infancy, spontaneous oral motility establishes a restricted set of movement primitives (e.g., a core set of movement patterns) that are later adapted and combined for early speech. ${ }^{1}$ Under these conditions, young children acquiring new speech sounds would modify and combine the existing set of movement primitives (Stark, 1980). Strong evidence for this hypothesis would be provided by the observation that spontaneous oral movements occur frequently in early postnatal development and that their kinematic features are similar each time they are observed.

Although spontaneous movements of the lips, tongue, and jaw appear to be abundant in early prenatal and postnatal development, little is known about their kine- matic characteristics, their developmental course, and their potential contribution to the acquisition of speech. Physiologic studies of prespeech oromotor behaviors are rare with the exception of investigations focused specifically on chewing (Green et al., 1997), sucking (Finan \& Barlow, 1998), and perioral reflexes (Barlow, Finan, \& Rowland, 1992). This investigation provides a comprehensive kinematic description of a subset of spontaneous orofacial movements - silent movements that appear to be undirected or exploratory rather than in response to environmental stimulation (i.e., imitative facial gestures or facial expressions), task demands (i.e., chewing and vocal communication), or auditory self-stimulation (i.e., extended vocal play).

The primary motivation for studying silent spontaneous movements is that developmental changes in these movements may reveal basic features of early oromotor control that influence the acquisition of specific movement patterns for chewing, sucking, and communication. Facial movements that were accompanied with vocal output, although potentially spontaneous, were excluded based on a prior finding showing them to be a fundamentally different category of movements than nonvocalized spontaneous facial movements. Specifically, the excursions of facial movements during vocal play are much larger than those produced silently, presumably because the infant is exploring the sound generating capabilities of their vocal instrument (Green \& Wilson, 2003). In addition, Holowka and Petitto (2002) showed that the lip movements of babbling are distinct from those associated with smiles. In their study, babbling was produced with right mouth asymmetry, nonbabbling was produced with symmetry between the left and right sides of the mouth, and smiling was produced with left mouth asymmetry. They interpreted the right mouth asymmetry in babbling to reflect the involvement of the language centers in the left cerebral hemisphere.

Additional motivation for studying silent facial movements comes from the findings of studies of spontaneous limb movements, which have been used to identify fetal distress and to predict a child's rate of motor skill development (Roberts, Griffin, Mooney, Cooper, \& Campbell, 1980). Knowledge about the typical development of spontaneous oral movements may have similar clinical implications particularly for determining the developmental status of nonverbal children and young children before they acquire speech.

Silent spontaneous orofacial movements were recorded in a cross-sectional sample of typically develop-

1 The existence of movement primitives in early oromotor development is attractive from the perspective that it would provide one possible explanation for the orderly emergence of sound acquisition and why children reared in different language environments exhibit similarities in early vocal development (Kent, 1984, 1992; Locke, 1983; Oller, Eilers, Urbano, \& Cobo-Lewis, 1997). Of course, learning and perfecting the motor skills for speech will also require auditory input and kinesthetic experiences that are specific to speech gestures. 
ing infants from 1 to 12 months of age using an optically based 3D motion capture system. Seven parameters of motor performance were derived from the facial kinematic signals: movement space, peak and average movement speed, movement epoch duration, path distance, spatial coupling, and temporal coupling. Data analyses were designed to test three hypotheses:

H1. The kinematics of spontaneous facial movements exhibit age-related changes during the first year of life,

H2. Developmental changes in motor performance are similar for different facial regions, and

H3. Early spontaneous facial movements are relatively stereotypic. A facial region will be considered to exhibit stereotyped movements, if its spatial and temporal characteristics are relatively consistent across epochs.

\section{Methods}

\section{Participants}

Facial motions were studied cross-sectionally from 29 typically developing infants at five ages: 1, 5, 7, 9, and 12 months. Twelve additional participants failed to complete the experiment primarily due to fussiness, sleepiness, or noncompliance with marker placement. The 1-month age group had five participants ( 1 female, 4 males) and the other age groups had six participants with the following female to male ratios: 5 months 3:3, 7 months $-2: 4,9$ months $-3: 3$, and 12 months $-3: 3$. Due to the small number of subjects in each group, sex differences were not investigated. Participants came from Standard American English speaking homes. Prior to inclusion in the study, each participant's caregiver was interviewed regarding the child's birth and health history, as well as their motor, cognitive, and communication developmental status. All participants were reported to have negative histories of developmental or neurological problems. To screen for possible developmental delays that were not apparent at the time of data collection, each caregiver was interviewed 6 months after the laboratory session regarding their child's motor, cognitive, and communication developmental status. All of the children originally enlisted in the study passed the postdata collection screener.

\section{Data Collection}

Infants were seated in an infant seat that was secured within a sound-reinforced chair (Starkey AEMII chair). The sound-reinforced chair was used to dampen ambient sounds (e.g., computer-fan noise) and to obstruct the view of objects on the side and behind the child, which encouraged them to direct their gaze toward the cameras. The entire data collection session was recorded using digital video and audio $\left(f_{\mathrm{s}}=44.1 \mathrm{kHz}\right)$. The audio channel was recorded using a lavaliere condenser microphone (AudioTechnicaAT831R) that was mounted to the interior of the sound-reinforced chair directly above the infants' face. A five-point support system was used to secure the infants in the seat and to provide adequate trunk support. For children 5 months and younger, the infant seat was slightly reclined and, when necessary, supports were used to stabilize the head and trunk. Silent facial movements were recorded during each data collection session, which lasted between 20 and $45 \mathrm{~min}$. Each session was tailored to ensure that the infant was content and involved a variety of activities including periods of silence without the parent in view, playing with manipulatives, watching a video of other infants, and verbal play. The order and duration of activities varied according to each child's interest.

\section{Identification of Spontaneous Movements}

Three trained transcribers identified silent spontaneous movement epochs from the full-face video recordings of each datacollection session. The objective of transcription was to identify facial movements that were not associated with specific functions, vocalization, speech, or environmental stimulation. Consequently, the transcriptionists were trained to identify spontaneous movements based on the following exclusionary criteria: lip and jaw movement in the absence of crying, smiling, grunting, coughing, yawning, sneezing, chewing, sucking, hiccups, vocalizing, touching, or speaking. Facial expressions such as smiling, frowning, and grimacing were also excluded. The remaining facial movements, which were the focus of this investigation, were silent spontaneous movements of the mouth (e.g., jaw opening or closing, lip spreading, lip protrusions, silent oscillations of the lips or jaw). Hooker (1944) and Humphrey (1970) used a similar, but more general, definition of spontaneous movements: motor activity for which the stimuli are unknown. Movement data were parsed into epochs that were separated by at least $500 \mathrm{~ms}$ (30 video frames) of no detectable facial motion on the full-face digital video recording.

Inter-rater reliability of epoch identification and transcription was assessed through the retranscription of 5 of the 29 data collection sessions. One subject in each group was arbitrarily selected for this analysis. Inter-rater agreement percentages for the transcription of identical data collections sessions ranged from $72 \%$ to $90 \%(M=81.75 \%, S D=6.17)$, which was judged as acceptable.

\section{Recording Facial Kinematics and Signal Conditioning}

Facial movements were tracked in three-dimensions using a video-based movement tracking system (Vicon 250, Vicon Motion Systems, 2000). This system tracked the motion of small reflective markers placed on the face at 60 frames per second using five infrared video cameras. The image-processing software provided by Vicon identified the marker locations in each two-dimensional infrared camera image to compute its $3 \mathrm{D}$ location relative to a calibration plate that was positioned in the data collection room. Prior to recording, lens distortions were corrected and all cameras were calibrated according to manufacturer specifications. Passive reflective markers, which were approximately $2 \mathrm{~mm}$ in diameter, were placed midline on the right and left eyebrows (ER and EL, respectively), upper lip (UL), lower lip (LL), and on the right and left oral commissures (CR and CL, respectively). In addition, three markers were placed on the chin, one on midline and inferior on the chin, and the other two approximately $1.5 \mathrm{~cm}$ to the left and right of the midline chin marker. Although there were three markers placed on the chin, only the chin marker located on the subject's right (JR) was studied. Marker JR was chosen to represent jaw motion because, based on prior findings from a 

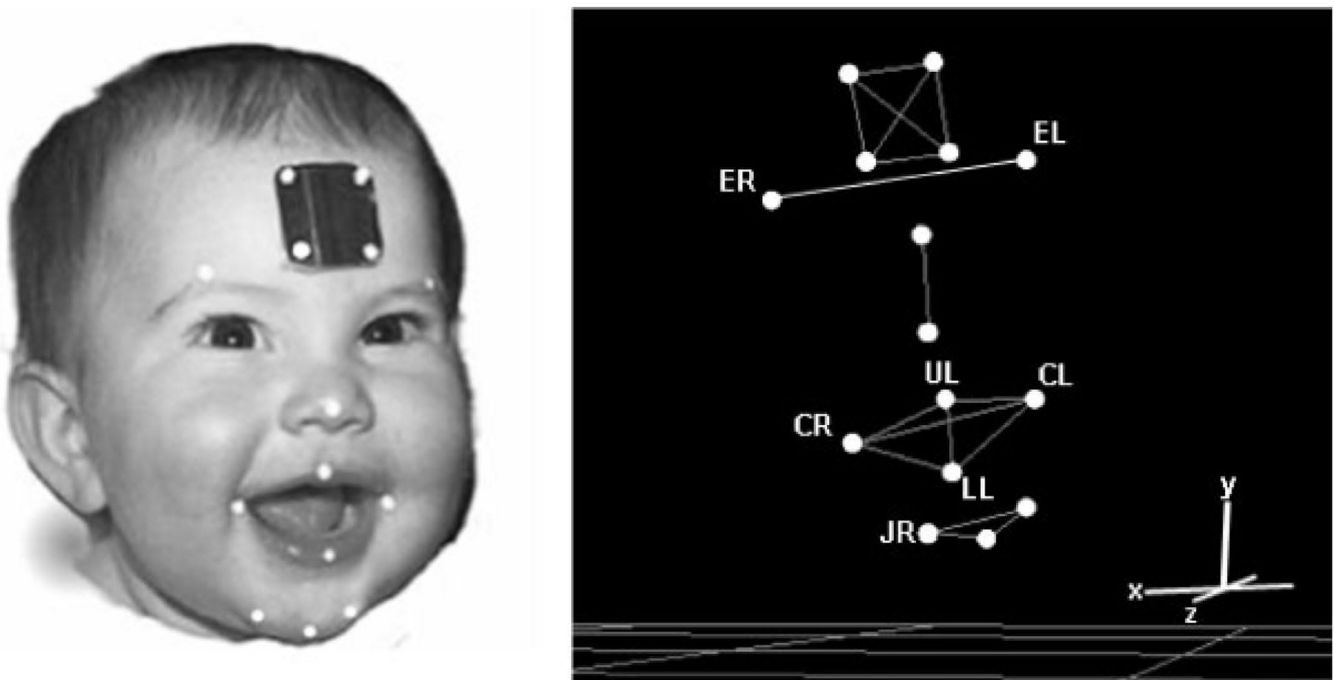

Figure 1. Left panel: A child fitted with the facial marker array. Right panel: 3D facial model reconstruction. For movement tracking, flat, circular reflective markers ( $2 \mathrm{~mm}$ in diameter) were placed on selected facial landmarks. The four reference markers placed on the forehead were used to correct for head movement that would otherwise be included in the facial movement signals. These four markers translate the origin to the head marker array and align the axis to the lines defined by these markers. Thus, all the facial kinematic data were expressed relative to the coordinate system defined by the four head makers. ER, right eyebrow; EL, left eyebrow; UL, upper lip; $\mathrm{CR}$, right oral commissure; CL, left oral commissure; LL, lower lip; JR, chin.

pilot study, lateral regions of the chin are less affected by lower lip motion than are markers placed on or near the midline. The facial marker array used for all subjects is displayed in Figure 1. All markers were flat except those located on the jaw. These $2 \mathrm{~mm}$ spheres were used on the chin to prevent drool from reducing marker reflectivity. Eyebrow markers were included to examine if the developmental course of the nonoral facial regions differed from that of the oral regions, which might be influenced by the abundance of motor experience gained from sucking, chewing, and vocalization.

To obtain movements of the facial markers that were independent of the head, the positions of the four forehead markers, which are displayed in Figure 1, were used to re-express the positions of the targeted facial markers in a head-based coordinate system. In this coordinate system, the origin was defined as the marker on the bottom right of the head marker array. The $y$ axis was defined by the upper right and lower right markers; the $x$ axis was defined by the bottom right and bottom left head markers; the $z$ axis was orthogonal to the $y$ and $x$ axis. Re-expression included the subtraction of the rotational and translational components of head motion. The lower lip marker represented the combined motions of the jaw and lower lip.

Following position tracking, the movement signals were digitally low-pass filtered $\left(f_{\mathrm{lp}}=10 \mathrm{~Hz}\right)$ using a zero-phase shift forward and reverse digital filter (Butterworth, 8 pole). An example, spontaneous movement epoch of the chin marker (JR) located on the subject's right is displayed in Figure 2.

\section{Parameters of Facial Motor Performance}

To enhance measurement reliability, automatic computer algorithms were developed to extract seven parameters of motor performance from each facial region (see Table 1). These algo- rithms were developed because the kinematic signals of spontaneous movements do not have easily identifiable landmarks for measurement. Each parameter was used to test for developmental changes in the characteristics of spontaneous facial movements. Variability of motor performance was also evaluated by computing the coefficient of variation for each kinematic parameter for each subject. If the defining characteristic of early facial movement is variation, then these parameters should be associated with high degrees of within variability across movement epochs.

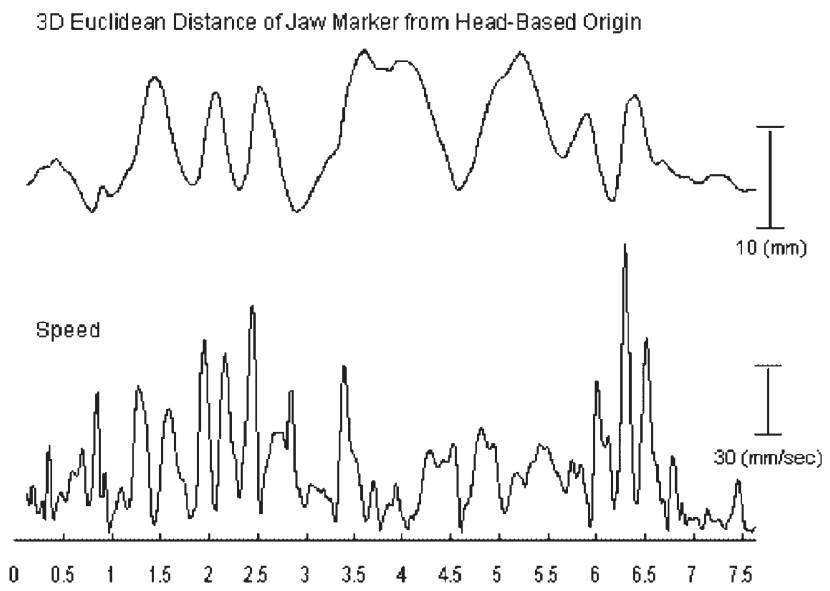

Time (s)

Figure 2. An example jaw kinematic trace recorded during a spontaneous movement epoch. The 3D distance time-history is based on the markers Euclidean distance from the head-based origin. Speed time-histories were derived by computing the first-order derivative of each marker's 3D distance time-history. 
Table 1. Seven Kinematic Parameters Were Used to Investigate Age-Related Changes in Spontaneous Movements

Kinematic Parameters

1. Movement space $\left(\mathrm{mm}^{3}\right)$

2. Average movement speed $(\mathrm{mm} / \mathrm{s})$

3. Peak movement speed $(\mathrm{mm} / \mathrm{s})$

4. Path distance ( $\mathrm{mm})$

5. Total movement epoch duration (ms)

6. Spatial coupling between markers (peak correlation coefficient)

7. Temporal coupling between markers (absolute and raw lag in $\mathrm{ms}$ )

3D Movement Space. To test for developmental changes in movement extent, a convex hull was fitted around each marker's 3D movement path (see Figure 3). The convex hull represents the smallest convex set containing all the points in the 3D motion path. The movement space that was occupied during each epoch was represented as the volume contained within each 3D convex hull fit. For each subject, this volumetric parameter of movement space was computed separately for each epoch rather than on the entire set of epochs to minimize the potential effects of outliers on the fit and to assess within subject variability across epochs.

Movement Speed. The average and peak movement speeds $(\mathrm{mm} / \mathrm{s})$ were measured from each movement epoch. These values were obtained from speed time-histories that were derived by computing the first-order derivative of each marker's Euclidean distance time-history (see Figure 2). Euclidean distances were relative to the head-marker based origin.

Movement Duration. The duration of each movement epoch was measured. To derive this parameter, the onset and the offset of facial movement was identified on the full-face digital video recordings of each subject as described previously in the Identification of Spontaneous Movements section.

Total Distance Traveled. The 3D distance that each marker traveled was computed by time-integrating each movement speed signal across each movement epoch.

Movement Coupling across Facial Regions. A cross-correlation analysis was performed to test for developmental changes in spatiotemporal coordination among different facial regions. The details of this analysis have been presented elsewhere (Green et al., 2000). Peak coefficients (negative or positive) and their associated lags were derived from the cross-correlation functions computed between the Euclidean distance time-histories of six marker pairs: $\mathrm{CR} \times \mathrm{CL}$, $\mathrm{CR} \times \mathrm{LL}, \mathrm{UL} \times \mathrm{LL}, \mathrm{UL} \times \mathrm{CR}, \mathrm{ER} \times \mathrm{EL}$, and $\mathrm{ER} \times \mathrm{LL}$. These pairs were selected to represent coupling relations between (1) orofacial regions (e.g., $U L \times L L, U L \times C R$ ), (2) symmetrical structures (i.e., ER $\times E L, C R \times C L$ ), and (3) nonorofacial and orofacial regions (i.e., ER $\times$ LL). Weak movement coupling

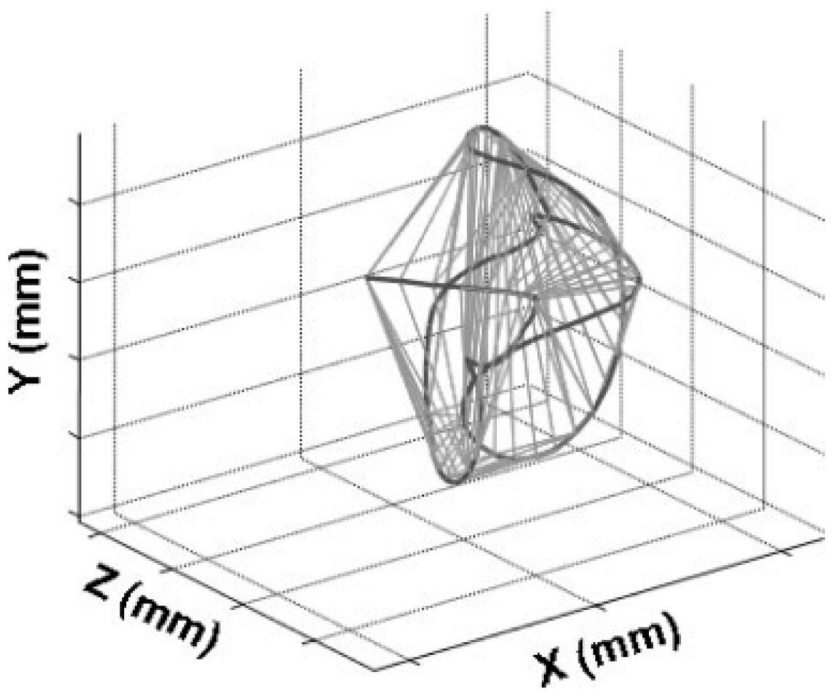

Figure 3. An example of the 3D movement space analysis performed on the jaw motion path obtained from a 7-month-old infant during a spontaneous movement.

was inferred from low peak cross-correlation coefficients and long lags; strong coupling was inferred from high crosscorrelation coefficients and short lags. Because this method inherently normalized inter-subject differences in movement magnitude, measured changes in movement coupling were independent of differences in anatomic size across subjects.

To simplify the interpretation of the correlation results, the upper lip signal was inverted so that its direction of movement during mouth opening and closing was the same as that of the jaw and lower lip. From each cross-correlation function, the most prominent peak (positive or negative) within a 200 ms window centered on zero lag was identified manually. Temporal resolution was determined by the videorecording rate (i.e., 60 frames per second). If the data analyst could not identify a peak in the cross-correlation function within the $200 \mathrm{~ms}$ window, the coefficient and lag for that articulatory pair were omitted from the final data corpus. Approximately $8 \%$ of all tokens were rejected by this criterion. This proportion did not differ significantly across age groups.

Lag times were analyzed in both their raw and absolute values, with each analysis having a different interpretation. Average raw values indicated the degree to which a bias existed in the temporal ordering of movements. That is, a long positive or negative average duration indicated that one of the markers in the pair typically moved before or after the other marker. In contrast, the absolute lag values indicated the degree of movement asynchrony regardless of the ordering.

\section{Statistical Treatment}

The values from each subject were collapsed and averaged for each kinematic parameter. Linear and nonlinear regression analyses were completed to test for developmental trends of each kinematic parameter. The coefficient data from the cross- 
Table 2. Results of Regression Analyses for Each Kinematic Parameter as a Function of Age and Facial Marker

\begin{tabular}{|c|c|c|c|c|c|c|}
\hline Performance Indices & Facial Marker & $R^{2}$ & B1 & B2 & $\mathrm{F}$ & $p$ \\
\hline 1. 3D movement space $\left(\mathrm{mm}^{3}\right)$ & EL & .18 & -.04 & & 4.80 & .038 \\
\hline \multirow[t]{6}{*}{ 2. Average speed (mm/s) } & EL & .18 & .23 & & 5.40 & .030 \\
\hline & UL & .58 & .61 & & 34.93 & .010 \\
\hline & CR & .23 & .56 & & 7.51 & .010 \\
\hline & CL & .66 & .78 & & 46.55 & .010 \\
\hline & LL & .40 & .77 & & 16.06 & .001 \\
\hline & JR & .47 & .82 & & 21.29 & .001 \\
\hline 3. Path distance (mm) & NS & & & & & \\
\hline 4. Duration (s) & All & .41 & -.56 & & 17.91 & .001 \\
\hline \multirow[t]{5}{*}{ 5. Coefficient $(r)$} & $\mathrm{ER} \times \mathrm{EL}$ & .27 & 1.67 & & 4.07 & .030 \\
\hline & $\mathrm{UL} \times \mathrm{CR}$ & .47 & .08 & & 20.53 & .000 \\
\hline & $\mathrm{UL} \times \mathrm{LL}$ & .23 & .07 & & 6.76 & .020 \\
\hline & $\mathrm{CR} \times \mathrm{CL}$ & .41 & .24 & .01 & 7.53 & .003 \\
\hline & $\mathrm{CR} \times \mathrm{LL}$ & .35 & .07 & & 12.53 & .002 \\
\hline \multirow[t]{2}{*}{ 6. Abs lag (ms) } & $\mathrm{CR} \times \mathrm{LL}$ & .33 & -.01 & & 11.42 & .003 \\
\hline & $\mathrm{UL} \times \mathrm{LL}$ & .32 & -.01 & & 10.62 & .003 \\
\hline 7. Raw lag (ms) & NS & & & & & \\
\hline
\end{tabular}

Only significant fits are displayed. NS indicates that the effect of age was not significant for all regions for that performance index; calculation for duration was based on the motion of all facial regions. ER, right eyebrow; EL, left eyebrow; UL, upper lip; CR, right oral commissure; $\mathrm{CL}$, left oral commissure; LL, lower lip; JR, chin.

correlation analysis were converted to Fisher's z-scores prior to statistical analysis. Repeated measures one-way ANOVAs and pairwise multiple comparison procedures using the Holm-Sidak method were performed to test for differences among facial regions across the measures. Figures are displayed for variables that showed age effects. Developmental changes in kinematic variability were also examined descriptively by computing the coefficient of variation for each subject for volume, distance, speed, and duration.

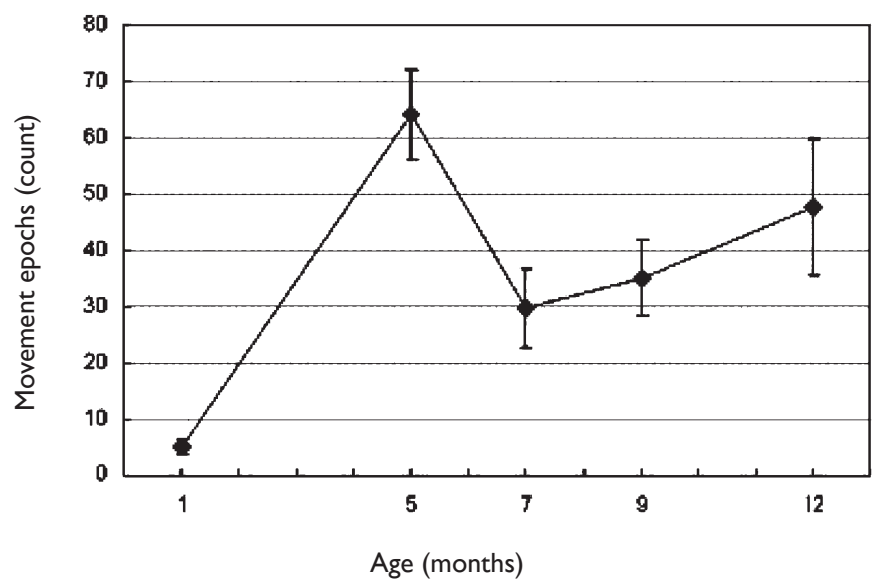

Figure 4. The average number of spontaneous movement epoch recorded at each age.

\section{Results}

The infants exhibited a large number of diverse spontaneous lip and jaw movements. The average number of epochs recorded across subjects within each age group is displayed in Figure 4. The number of spontaneous movements that were recorded increased significantly with age $(F[4,24]=6.79, p<.001)$. A transient peak in the number of spontaneous movements produced was observed in the 5 months old. The number of movements that could be studied from each subject depended on the number of facial movements that were produced, the session duration, and the number of movements that could be accurately captured using the five camera motion-capture system. Occasionally a movement was missed when a child turned away from the cameras.

Despite variability among trials, sessions, and infants, age-related trends in kinematics were observed for all of the facial regions. The average values for each kinematic parameter for orofacial region and age are displayed in Figures 5-8. For ease of visual interpretation, only the following markers were displayed in the Figures: ER, UL, CR, LL, JR. In addition, the average coefficient of variation $(\mathrm{CV})$ for each subject is displayed in the bottom panel of each Figure. These Figures are intended to provide information about developmental changes in performance variability associated with each kinematic parameter. The results of the regression analyses performed on each variable are reported in Table 2. 

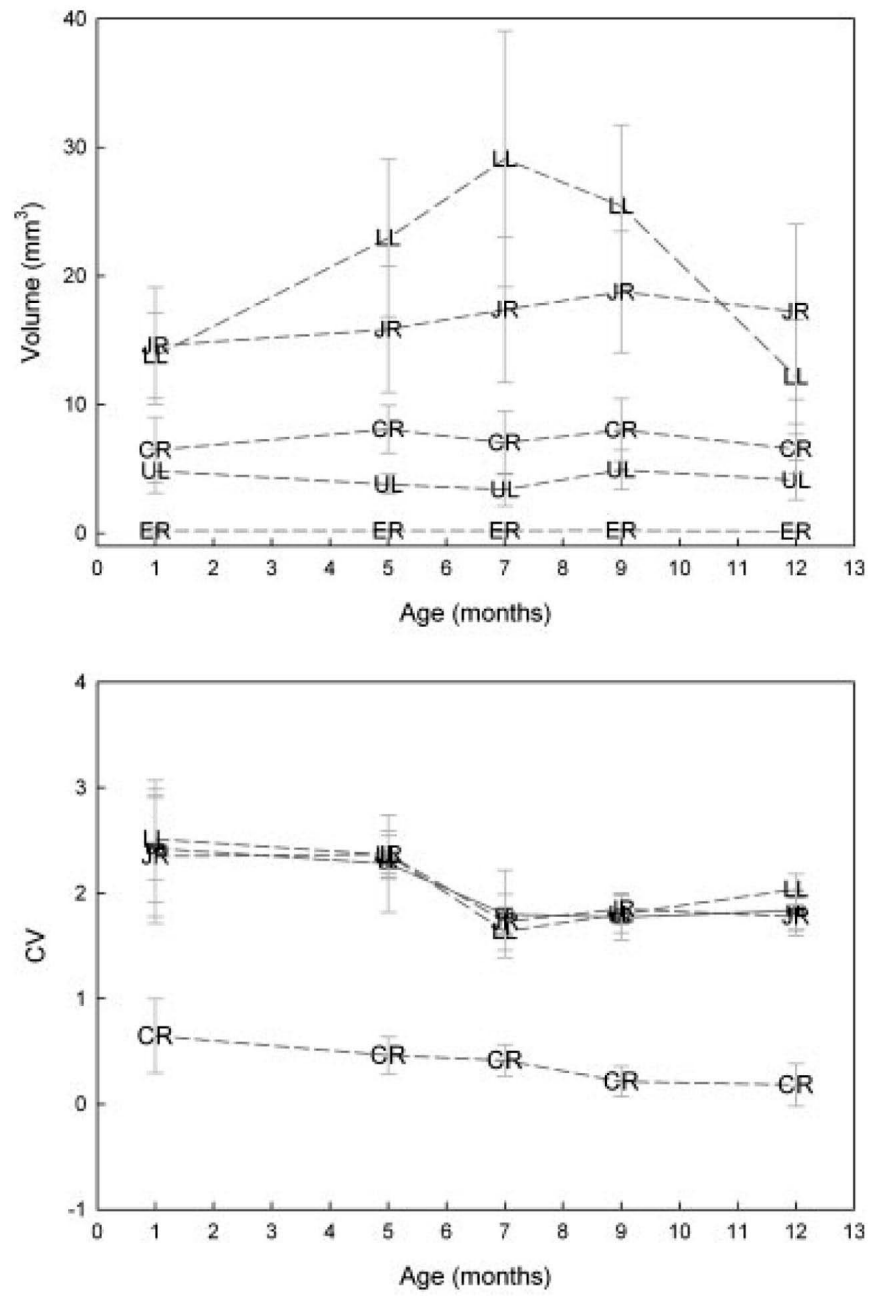

Figure 5. Top panel: Age-related changes in movement space for selected facial markers. Standard error bars represent across subject variation in each age group. Bottom panel: Agerelated changes in the within variability in movement space. ER, right eyebrow; UL, upper lip; CR, right oral commissure; LL, lower lip; JR, chin.

\section{D Movement Space}

Figure 5 contains the average 3D movement space observed for each facial region as a function of age. Significant age effects in movement space were not observed, though visual inspection of the lower lip marker data suggest a transitory increase in movement space at 7 months of age. Movement space differed significantly across markers except for the right and left eyebrow, the right and left commissures, the lower lip, and the jaw. Specifically, the movements of the lower lip and jaw were significantly larger than those of all other facial regions ( $p<.01$, for all comparisons). Eyebrow movements were significantly smaller than those observed for all other regions ( $p<.01$, for all comparisons); at all ages, movement of the eyebrows was negligible.
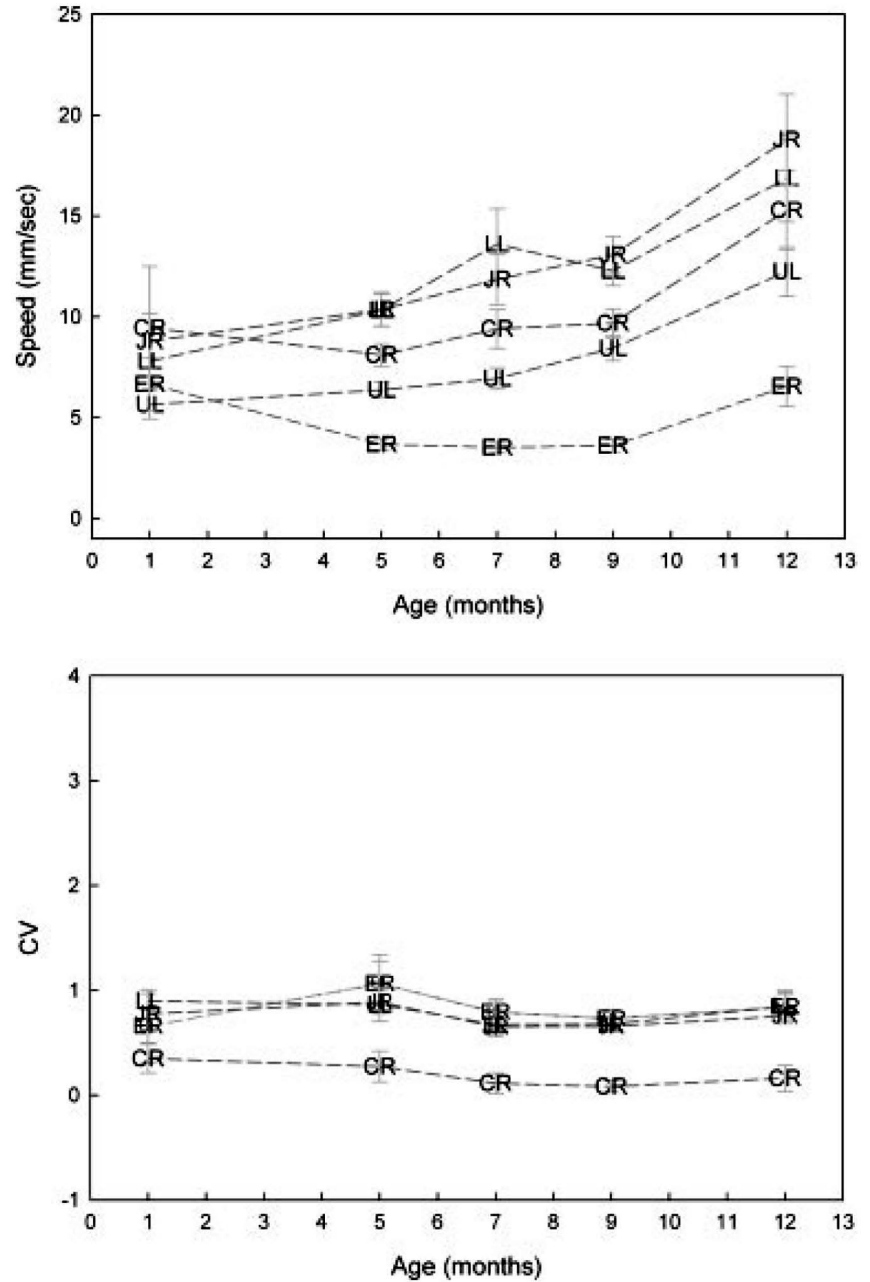

Figure 6. Top panel: Age-related changes in average movement speed for selected facial markers. Standard error bars represent across subject variation in each age group. Bottom panel: Age-related changes in the within variability in average movement speed. ER, right eyebrow; UL, upper lip; CR, right oral commissure; LL, lower lip; JR, chin.

The CV plot in the bottom panel of Figure 5 shows that within variability was relatively large (i.e., greater than 1) for movement space. With the exception of the right eyebrow, age-related changes in $\mathrm{CV}$ values were remarkably similar across markers. The decrease in $\mathrm{CV}$ values was notable between ages 5 and 7 months.

\section{Movement Speed}

The average and peak speed of motion increased significantly with age except for the ER marker (see Table 2 and Figure 6). A linear model provided the best fit for all facial regions. The age trends for average speed were similar to those observed for peak speed, and therefore only the results for average speed are considered. Across markers, the developmental trajectory for average speed 


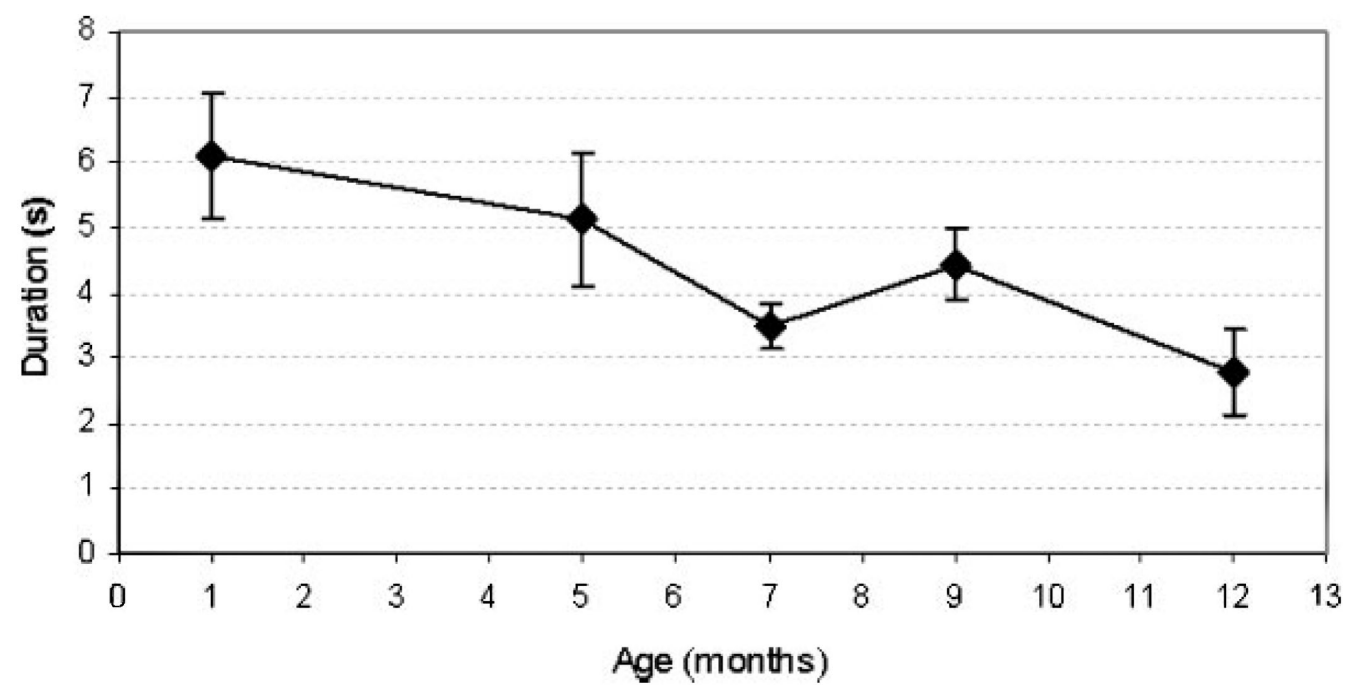

Figure 7. Age-related changes in movement epoch duration. Standard error bars represent across subject variation in each age group.

appeared to be similar. The CV data in the bottom panel of Figure 6 suggest that within variability ranged approximately between .6 and 1.1 for average speed. The developmental trajectory for $\mathrm{CV}$ values was also similar across markers.
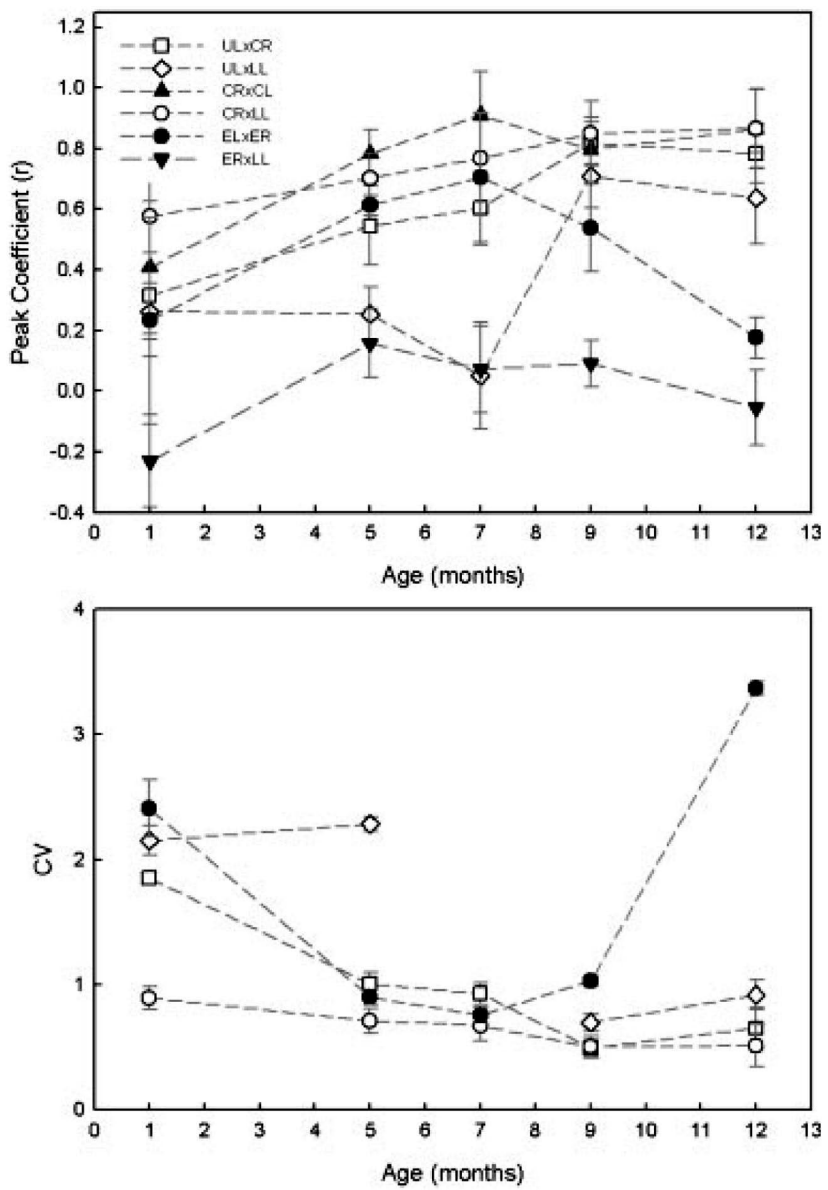

Movement speed differed significantly among all markers except for the following pairs: right and left eyebrow, the right and the left commissures, and the lower lip and jaw. The movement speed of the lower lip and jaw were significantly greater than those of all other facial regions $(p<.01$, for all). The movement speeds of both commissures were greater than those for the upper lip ( $p<.01$ for both comparisons).

\section{Path Distance}

Path distance comparisons across ages revealed no statistically significant differences. Across all ages, the following average path distances and standard deviations were observed: right eyebrow $=8.08 \mathrm{~mm}$ (3.77), left eyebrow $=9.25 \mathrm{~mm}$ (4.52), upper lip $=15.06 \mathrm{~mm}$ (6.85); right commissure $=16.41 \mathrm{~mm}$ (6.0); left commissure $=17.43 \mathrm{~mm}$ (7.93); lower lip = $20.64 \mathrm{~mm}(7.68)$; jaw $=23.50 \mathrm{~mm}$ (11.35). Path distance differed significantly among all markers except for the following pairs: right and left eyebrow, right and left commissures, both commissures and the upper lip, and the lower lip and jaw. The path distance of the lower lip and jaw was significantly longer than those of all other facial regions $(p<$ .01 , for all comparisons). The path distance of the eye-

Figure 8. Top panel: Age-related changes in movement coupling between selected facial markers. Standard error bars represent across subject variation in each age group. Bottom panel: Age-related changes in the within variability in movement coupling. The $\mathrm{CV}$ values for $\mathrm{ER} \times \mathrm{LL}$ at all ages studied and for UL $\times$ LL at 7 months were very large (e.g., 11.5) and therefore not displayed. ER, right eyebrow; EL, left eyebrow; $\mathrm{UL}$, upper lip; CR, right oral commissure; CL, left oral commissure; LL, lower lip; JR, chin. 
brows was significantly shorter than those observed for all other regions ( $p<.01$, for all comparisons).

\section{Movement Epoch Duration}

The average duration of each movement epoch decreased significantly from 6.08 to $2.78 \mathrm{~s}$ between 1 to 12 months of age (see Table 2 and Figure 7).

\section{Interarticulator Coupling}

Average coefficient values for each age are displayed for selected pairs in Figure 8. Spatial coupling increased significantly for all pairs except ER $\times$ LL with age (see Table 2). A repeated measures ANOVA revealed a main effect for marker pairs for coefficient $(F[25,13]=44.66, p$ $<.001$ ). Pairwise comparisons were not performed due to the large number of comparisons. Across ages, coefficient values for articulatory pairs ranked from highest (i.e., strongest coupling) to lowest (i.e., weakest coupling) in the following order: $\mathrm{CR} \times \mathrm{CL}, \mathrm{CR} \times \mathrm{LL}, \mathrm{UL} \times \mathrm{CR}$, $\mathrm{ER} \times \mathrm{EL}, \mathrm{UL} \times \mathrm{LL}, \mathrm{ER} \times \mathrm{LL}$.

The absolute lag between the movements of different facial regions tended to be short ranging from 23 to 145 ms $(M=95)$. A repeated measures ANOVA revealed a significant main effect for marker pairs for absolute lag $(F[25,13]=44.66, p<.001)$. Due to the large number of comparisons, pairwise comparisons were also not performed on the absolute lag data. Across ages, lag values for articulatory pairs ranked from shortest to longest in the following order: $\mathrm{CR} \times \mathrm{CL}, \mathrm{CR} \times \mathrm{LL}, \mathrm{ER} \times \mathrm{EL}, \mathrm{UL} \times \mathrm{CR}$, $\mathrm{UL} \times \mathrm{LL}, \mathrm{ER} \times \mathrm{LL}$.

For all facial marker pairs, the effect of age on raw lag values were not statistically significant. In general, the average raw lag values approximated zero for all pairs, although, at 1 month of age, these values were varied considerably across facial regions and subjects.

\section{Discussion}

All of the infants in this study exhibited spontaneous facial movements. The number of spontaneous facial movements recorded during each data collection session increased with age. Collectively, the results of the kinematic analyses suggest that early spontaneous facial movements undergo significant developmental change during the first year of life. Although there were large individual differences in movement characteristics, several age effects were detected: the speed of movement increased, the duration of movement epochs decreased, and the coupling of movement among different facial regions increased. In general, the movements of most facial regions tended to be highly coupled at all ages. The observation of developmental trends in kinematics is re- markable given that spatial and temporal characteristics of these movements are free to vary (i.e., not governed by task demands such as those required for chewing, sucking or speaking). Evidence for stereotypic movements was not strong because the spatiotemporal characteristics of movement varied considerably across epochs. The present findings give the overall impression that spontaneous orofacial movements undergo significant developmental change in the direction of skill development for speech.

\section{Age-Related Changes Were Consistent with Those Observed during the Development of Speech and Limb Motor Systems}

The gradual increase observed in movement speed with age is consistent with several previous reports showing that articulatory speed and speech rate increase with age (Goffman \& Smith, 1999; Smith \& Gartenberg, 1984; Walker, Archibald, Cherniak, \& Fish, 1992). Based on visual inspection of Figure 6, the transition between 9 and 12 months of age was marked by a relatively large increase in average speeds, and lower lip and jaw kinematics appeared to become more variable across subjects. It is notable that these changes occurred at the time at which most infants begin to acquire their first spoken words (Dale \& Fenson, 1996).

The observation of slow movement speeds in early spontaneous orofacial movements raises the possibility that the rate at which children produce speech is limited by characteristics of the immature motor system such as a relatively slow nerve conduction time. Using transcranial magnetic stimulation (TMS), Müller and Hömberg (1992) showed that the rate of performance of upper limb movements during fastest repetitive tapping, aiming, and pegboard tasks in children were closely associated with the developmental slope of the fastest cortico-motoneuronal efferents. In early development, nerve conduction velocity becomes faster because of increases in axon diameter and myelin thickness as well as changing properties of the cell membrane (Waxman, 1980). TMS of motor cortex in children suggest that the fastest corticospinal efferents do not reach adult conduction times until the age of 8-10 years (Müller, Hömberg, \& Lenard, 1991). The dependence on afferent feedback and limitations in cognitive processing may also be important factors in determining the rate at which speech is produced at different stages of development (Smith \& McLean-Muse, 1986).

The number of spontaneous movements increased sharply at 5 months of age, which is just prior to when children typically begin to produce canonical babble (Oller, 1980; Stark, 1980) and stereotypies in the jaw, hands, and arms (Ejiri, 1998; Meier, McGarvin, Za- 
kia, \& Willerman, 1997; Thelen, 1979). Further work is needed to determine if the neuromotor mechanisms that are responsible for these changes in spontaneous orofacial movements are the same as those that trigger the emergence of babble, which is a developmental achievement that ostensibly marks the beginnings of adult-like speech (Kent, 1984; Stark, 1980).

Movement space and path distance did not differ across age groups, whereas other parameters showed strong developmental trends. An increase in movement space and path distance might have been expected, particularly with growth of the mandible. Several other investigators have also reported that lip and jaw displacements during speech do not change substantially with age and growth (Goffman \& Smith, 1999; Riely \& Smith, 2003; Smith \& Gartenberg, 1984).

The results of the cross-correlation analysis suggested that the different parts of the mouth moved synchronously at all ages. Symmetry and, particularly, synchrony may be a general characteristic of immature motor control that influences the development of speech production and other skilled behaviors. For example, bilateral arm movements also exhibit a similar bias toward symmetry in early development (Fagard \& Jacquet, 1989). Similarly, movements of the upper and lower lips also appear to be highly coupled in early childhood (Green et al., 2000). This very restrictive form of lip coordination may limit the sound producing capabilities of young children and suggest that further gains in speech will require an increase in the ability to move the upper and lower lips independently (Green et al., 2000).

\section{Age-Related Changes Were Relatively Similar among Facial Regions}

In the present study, we examined whether age-related changes in the kinematics of spontaneous movements were distinct across facial regions. This question was of interest because previous findings suggested that jaw movement patterns develop much earlier than do those of the lips (Green, Moore, \& Reilly, 2002). This finding led to the conclusion that characteristics of the immature oromotor control has a strong influence on the order that new sounds are acquired by young children (Green et al., 2002). The present data offer some insight into whether such developmental biases in oromotor development are apparent before children acquire speech.

Visual inspection of the growth functions in many of the Figures gives the overall impression that age-related changes appeared to be similar across different facial regions, though some subtle differences were noted, particularly in the correlation analysis. Coupling among most facial regions was strong at all ages, whereas up- per and lower lip coupling was very weak until 7 months, when coupling increased sharply. In addition, right and left eyebrow coupling decreased sharply after 7 months. Only the nonorofacial region, the right eyebrow, showed this distinct developmental path for coupling suggesting that the neural centers for lower face control are undergoing unique changes, perhaps related to specific experiences such as those obtained by the jaw and lips during sucking, chewing, and early vocalization. On the other hand, for many kinematic parameters, eyebrow movement exhibited similar developmental changes as oral regions, suggesting that the observed age-related changes are independent of such specific experience.

\section{Stereotypy in Facial Movements Were Not Evident}

The suggestion that stereotypic behaviors form the basis from which skilled motor behaviors, such as speech develop, has great parsimony - these behaviors could form a restricted set of stable movement patterns that could be altered and combined during the acquisition of motor control. Presumably, stereotypic movements would be evidenced by relatively invariant kinematic characteristics across epochs. One feature of orofacial coordination that appeared to be relatively consistent across movement epochs was the tendency for different facial regions to move synchronously and symmetrically. The majority of the present findings suggest, however, that the spatial and temporal characteristics of early orofacial movements are not patterned, but highly variable. The average $\mathrm{CV}$ values for working space were particularly high, ranging from 1.6 to 2.5; coefficient of variation values greater than 1 were interpreted to suggest that facial movements were highly variable. Within subject variability for movement speed and distance was also high, but lower than that observed for working space.

In contrast, the findings from several other kinematic-based studies have reported that early spontaneous movements exhibit a remarkable degree of spatial and temporal organization. Thelen (1985) reported that early spontaneous leg movements are highly patterned in time and space. Heriza (1988) observed supine kicking of premature infants to be highly coordinated, but different in form from the adult (Heriza, 1988). Highly coordinated jaw and tongue oscillations have also been observed in the fetus (de Vries et al., 1985).

The suggestion that stereotypies serve an important function in early motor development becomes less appealing if variability in early motor performance is the rule and not the exception. The absence of stereotyped facial movements does not, however, eliminate the possibility of stereotypy at the level of muscle activation. 
Specifically, coordinated muscle activity may not always result in well-controlled movements in early development because infants are learning to manage interactions between the active forces generated by their muscles and the passive forces of their musculoskeletal system (Green et al., 1997, 2000).

\section{Experimental Limitations}

Although the present study focused on facial movements that were only unintentional or exploratory, some of the movements studied may have been directed toward the caregiver or contained subtle emotional overlays that were difficult to detect; in practice, determining the intention of facial movements from infants can be difficult, even when stringent exclusionary criteria are implemented. In addition, while these data provide insights into the overall direction of developmental change in facial movement, longitudinal studies are needed to resolve individual growth curves in the development of facial motor control. Another limitation was weak statistical power, which was due to the small number of subjects and the large amount of performance variability across subject within each age group. A larger number of subjects will need to be studied once motion capture and data reduction technologies become more automated. Finally, because the skin of lower lip is contiguous with that of the chin, elevation of the lower lip can stretch the skin covering the chin, particularly on the region directly below midline lower lip. As discussed previously, an attempt was made to minimize this effect by using the chin marker that was off midline, away from the fleshy part of the chin.

\section{Conclusions}

These data give the overall impression that spontaneous activity of orofacial structures is a hallmark feature of early oromotor development. Although these movements are undirected, they undergo significant developmental change. Moreover, some of the developmental changes in kinematics are in the direction of developing skills for speech. The present findings suggest that spontaneous movements of the face are highly variable and rarely patterned. The observation of variability does not diminish the potential role of oral spontaneous movements for speech. In fact, performance variability may be an essential developmental characteristic required for establishing a full repertoire of movement patterns and the sensorimotor systems that support speech. Future work will be directed toward examining if these spontaneous movements exhibit developmental continuity with the emerging gestures of early speech. In addition, the present findings motivate further inquiry into the use of movement speed during spontaneous movements as a clinical indicator of oromotor maturation. This parameter might be particularly well suited to identify preverbal children who are at risk of communication impairments. Future work is needed to specify the anatomic and physiologic significance of spontaneous orofacial movement on the development of speech.

\section{References}

Barlow, S. M., Finan, D. S., \& Rowland, S. G. (1992). Mechanically evoked perioral reflexes in infants. Brain Research, 599, 158-160.

Bekoff, A. (1986). Ontogeny of chicken motor behaviors: Evidence for multiuse limb pattern generating circuitry. In S. Grillner, P. S. G. Stein, D. G. Stuart, H. Forssberg, \& R. M. Herman (Eds.), Neurobiology of vertebrate locomotion (pp. 433-453). Hampshire, England: Macmillan Press.

Bekoff, A. (2001). Spontaneous embryonic motility: An enduring legacy. International Journal of Developmental Neuroscience, 19, 155-160.

Birnholz, J. C., Stephens, J. C., \& Faria, M. (1978). Fetal movement patterns: A possible means of defining neurologic developmental milestones in utero. American Journal of Roentgenology, 130, 537-540.

Coghill, G. E. (1929). Anatomy and the problem of behaviour. New York: Cambridge University Press.

Dale, P. S., \& Fenson, L. (1996). Lexical development norms for young children. Behavioral Research Methods, Instruments \& Computers, 28, 125-127.

de Vries, J. I. P., Visser, G. H. A., \& Prechtl, H. F. R. (1982). The emergence of fetal behaviour I: Qualitative aspects. Early Human Development, 7, 301-322.

de Vries, J. I. P., Visser, G. H. A., \& Prechtl, H. F. R. (1985). The emergence of fetal behaviour II: Qualitative aspects. Early Human Development, 12, 99-120.

Edelman, G. M. (1987). Neural Darwinism: The theory of neuronal group selection. New York: Basic Books.

Ejiri, K. (1998). Relationship between rhythmic behavior and canonical babbling in infant vocal development. Phonetica, 55, 226-237.

Fagard, J., \& Jacquet, A. (1989). Onset of bimanual coordination and symmetry versus asymmetry of movement. Infant Behavior and Development, 12, 229-235.

Fields, R. D., \& Nelson, P. G. (1992). Activity-dependent development of the vertebrate nervous system. International Review of Neurobiology, 34, 133-214.

Finan, D. S., \& Barlow, S. M. (1998). Intrinsic dynamics and mechanosensory modulation of nonnutritive sucking in human infants. Early Human Development, 52, 181- 197. 
Geerdink, J. J., Hopkins, B., Beek, W. J., \& Heriza, C. B. (1996). The organization of leg movements in preterm and full-term infants after term age. Developmental Psychobiology, 29, 335-351.

Goffman, L., \& Smith, A. (1999). Development and phonetic differentiation of speech movement patterns. Journal of Experimental Psychology: Human Perception \& Performance, 25, 649-660.

Gottlieb, G. (1992). Individual development and evolution: The genesis of novel behavior. New York: Oxford Press.

Green, J. R., Moore, C. A., Higashikawa, M., \& Steeve, R. W. (2000). The physiologic development of speech motor control: Lip and jaw coordination. Journal of Speech, Language, \& Hearing Research, 43, 239-255.

Green, J. R., Moore, C. A., \& Reilly, K. J. (2002). The sequential development of jaw and lip control for speech. Journal of Speech, Language, and Hearing Research, 45, 66-79.

Green, J. R., Moore, C. A., Ruark, J. L., Rodda, P. R., Morvee, W. T., \& Van Witzenburg, M. J. (1997). Development of chewing in children from 12 to 48 months: Longitudinal study of EMG patterns. Journal of Neurophysiology, 77, 2704-2716.

Green, J. R., \& Wilson, E. M. (2003). The significance of spontaneous orofacial motility in early vocal development. Proceeding of the Conference on Progress in Motor Control IV: Motor Control and Learning over the Life Span. Université de Caen Basse-Normandie (France).

Gust, J., Wright, J. J., Pratt, E. B., \& Bosma, M. M. (2003). Development of synchronized activity of cranial motor neurons in the segmented embryonic mouse hindbrain. The Journal of Physiology, 550, 123-133.

Hall, B. K., \& Herring, S. W. (1990). Paralysis and growth of the musculoskeletal system in the embryonic chick. Journal of Morphology, 206, 45-56.

Hall, W. G., \& Oppenheim, R. W. (1987). Developmental psychobiology: Prenatal, perinatal, and early postnatal aspects of behavioral development. Annual Review of Psychology, 38, 91-128.

Hamburger, V., Wenger, E., \& Oppenheim, R. (1966). Motility in the chick embryo in the absence of sensory input. Journal of Experimental Zoology, 162, 133-159.

Heriza, C. B. (1988). Organization of leg movements in preterm infants. Physical Therapy, 68, 1340-1346.

Herring, S. W. (1985). The ontogeny of mammalian mastication. American Zoology, 25, 339-349.

Holowka, S., \& Petitto, L. A. (2002). Left hemisphere cerebral specialization for babies while babbling. Science, 297, 1515.

Hooker, D. (1944). The origin of overt behavior. Ann Arbor, MI: University of Michigan.

Humphrey, T. (1970). The development of human fetal ac- tivity and its relation to postnatal behavior. Advances in Child Development \& Behavior, 5, 1-57.

Humphrey, T. (1971). Development of oral and facial motor mechanisms in human fetuses and their relation to craniofacial growth. Journal of Dental Research, 50, 1428- 1441.

Kalb, R. G., \& Hockfield, S. (1992). Activity-dependent development of spinal cord motor neurons. Brain Research, 17, 283-289.

Kent, R. D. (1984). Psychobiology of speech development: Coemergence of language and a movement system. American Journal of Physiology, 246, 888-894.

Kent, R. D. (1992). The biology of phonologic development. In C. A. Ferguson, L. Menn, \& C. Stoel-Gammon (Eds.), Phonological development: Models, research, implications (pp. 65-90). Timonium, MD: York Press.

Kuo, Z. (1967). The dynamics of behavioral development. New York: Random House.

Landmesser, L. T., \& Morris, D. G. (1975). The development of functional innervation in the hind limb of the chick embryo. The Journal of Physiology, 249, 301-326.

Locke, J. L. (1983). Phonological Acquisition and Change. San Diego: Academic Press.

Meier, R. P., McGarvin, L., Zakia, R. A., \& Willerman, R. (1997). Silent mandibular oscillations in vocal babbling. Phonetica, 54, 153-171.

Müller, K., \& Hömberg, V. (1992). Development of speed of repetitive movements in children is determined by structural changes in corticospinal efferents. Neuroscience Letters, 144, 57-60.

Müller, K., Hömberg, V., \& Lenard, H. G. (1991). Magnetic stimulation of motor cortex and nerve roots in children: Maturation of cortico-motoneuronal projections. Electroencephalography and Clinical Neurophysiology, 8, 163-170.

Oller, D. K. (1980). The emergence of the sounds of speech in infancy. In G. H. Yeni-Komshian, C. A. Ferguson, \& J. Kavanagh (Eds.), Child phonology: Production (pp. 93112). New York: Academic Press.

Oller, D. K., Eilers, R. E., Urbano, R., \& Cobo-Lewis, A. B. (1997). Development of precursors to speech in infants exposed to two languages. Journal of Child Language, $24,407-425$.

Oppenheim, R. W., Pittman, R., Gray, M., \& Maderdrut, J. L. (1978). Embryonic behavior, hatching, and neuromuscular development in the chick following a transient reduction of spontaneous motility and sensory input by neuromuscular blocking agents. Journal of Comparative Neurology, 179, 619-640.

Riely, R. R., \& Smith, A. (2003). Speech movements do not scale by orofacial structure size. Journal of Applied Physiology, 96, 2119-2126.

Roberts, A. B., Griffin, D., Mooney, R., Cooper, D. J., \& Campbell, S. (1980). Fetal activity in 100 normal third 
trimester pregnancies. Journal of Obstetrics and Gynecology Research, 87, 480-484.

Roeder, K. D. (1963). Nerve cells and insect behavior. Cambridge, MA: Harvard University Press.

Smith, B. L., \& Gartenberg, T. E. (1984). Initial observations concerning developmental characteristics of labio-mandibular kinematics. Journal of the Acoustical Society of America, 75, 1599-1605.

Smith, B. L., \& McLean-Muse, A. (1986). Articulatory movement characteristics of labial consonant productions of children and adults. Journal of the Acoustical Society of America, 80, 1321-1328.

Stark, R. E. (1980). Stages of speech development in the first year of life. In G. Yani-Komshian, J. Kavanagh, \& C. A. Ferguson (Eds.), Child Phonology? (pp. 73-92). New York: Academic Press.

Thelen, E. (1979). Rhythmical stereotypies in normal human infants. Animal Behaviour, 27, 699-715. Spontaneous Facial Motility 27
Thelen, E. (1985). Developmental origins of motor coordination: Leg movements in human infants. Developmental Psychobiology, 18, 1-22.

Thelen, E. (1995). Motor development: A new synthesis. American Psychologist, 50, 79-95.

Thelen, E., \& Fisher, D. M. (1983). From spontaneous to instrumental behavior: Kinematic analysis of movement changes during very early learning. Child Development, 54, 129-140.

Walker, J. F., Archibald, L. M., Cherniak, S. R., \& Fish, V. G. (1992). Articulation rate in 3- and 5-year-old children. Journal of Speech Hearing Research, 35, 4-13.

Waxman, S. G. (1980). Determinants of conduction velocity in myelinated nerve fibers. Muscle and Nerve, 3, 141-150. 